\title{
Luminescence of Highly Photoexcited GaN Epilayers and Heterostructures Grown on Different Sapphire Crystal Planes
}

S. JURŠĖnas ${ }^{a, *}$, S. Miasojedovas ${ }^{a}$, G. KURILČIK $^{a}$, V. LiUolia ${ }^{a}$, A. ŽUnauskas ${ }^{a}$, C.Q. Chen $^{b}$, J.W. YANG ${ }^{b}$, E. KuOKŠTis ${ }^{b}$, V. Adivarahan ${ }^{b}$ AND M. AsIF $\mathrm{KHAN}^{b}$

${ }^{a}$ Institute of Materials Science and Applied Research, Vilnius University Sauletekio 9-III, 10222 Vilnius, Lithuania

${ }^{b}$ Department of Electrical Engineering, University of South Carolina Columbia, SC 29208, USA

GaN epilayers and AlGaN/GaN multiple quantum wells grown by metalorganic chemical vapor deposition on different crystal planes $(c, a$, and $r$ ) of the sapphire substrate were studied by excitation intensity dependent and time-resolved photoluminescence. In polar multiple quantum wells grown on $a$ - and $c$-planes, a blueshift of the luminescence band with increasing the excitation energy was observed, indicating that screening of built-in field by free carriers takes place, whereas in nonpolar $r$-plane grown multiple quantum wells, the luminescence band maintained an almost constant peak position. Full screening of built-in field was achieved at the excitation densities higher than $0.3 \mathrm{~mJ} / \mathrm{cm}^{2}$. Under conditions of screened built-in electric field the structures were characterized by carrier lifetime. It was shown that nonpolar multiple quantum wells suffer from high density of nonradiative traps that can be due to substrate related threading dislocations.

PACS numbers: 78.55.Cr, 73.21.Fg, 72.20.Jv, 78.47.+p

\section{Introduction}

III-nitride semiconductors are materials of high interest, which can be successfully applied for developing of blue and UV optoelectronic devices such as light emitting and laser diodes, as well as high-voltage and high-power electronic devices [1]. However, these applications are impeded by built-in electric fields, which can

*corresponding author; e-mail: saulius.jursenas@ff.vu.lt 
be reduced by growing nonpolar structures, for instance, growing on the $r$-plane of the sapphire substrate [2-6].

In the present paper luminescence properties of $\mathrm{GaN}$ epilayers and AlGaN/GaN multiple quantum wells (MQWs) grown over $c_{-}\{0001\}, a^{-}\{11-20\}$ and $r$ - $\{1-102\}$ planes of sapphire substrate are presented. High excitation intensity experimental conditions applied, made it possible to characterize the material of both polar and nonpolar MQWs and corresponding substrates by time-resolved photoluminescence.

\section{Experimental}

The $c$-plane GaN epilayers were grown on $c$-plane and $a$-plane sapphire substrates using a low-pressure metalorganic chemical vapor deposition reactor. A $1.5 \mu \mathrm{m}$ thick GaN epilayer was deposited on the sapphire substrate with a $20 \mathrm{~nm}$ thick low-temperature GaN buffer layer. The $a$-plane GaN epilayer was grown over the $r$-plane sapphire substrates using a similar two-step growth procedure. The thickness of GaN epilayer was around $1.5 \mu \mathrm{m}$. Additionally, three MQW samples were prepared. 5 MQWs, consisting of $5 \mathrm{~nm}$ GaN quantum wells and $8 \mathrm{~nm}$ AlGaN barriers (approximately $25 \% \mathrm{Al}$ ) pairs were grown on GaN epilayer that was deposited over $c^{-}, a$-, and $r$-planes of the sapphire substrates. The orientation of the grown structures were proved by X-ray diffraction measurements [5, 6].

The samples were excited by the fourth harmonic (photon energy $h \nu_{\mathrm{g}}=$ $4.66 \mathrm{eV}$ ) of the actively-passively mode-locked YAG: $\mathrm{Nd}^{3+}$ laser (pulse duration was $20 \mathrm{ps}$, repetition rate was $2.7 \mathrm{~Hz}$, and maximum pump energy was $40 \mu \mathrm{J}$ ). The size of the excitation spot was approximately $1 \mathrm{~mm}$. Photoluminescence was collected in backscattering geometry and dispersed by a $0.4 \mathrm{~m}$ grating monochromator. Toluene optical Kerr shutter was used for temporal resolution (20 ps) of the luminescence decay. The experiments were carried out at room temperature.

\section{Results and discussion}

Figure 1 displays time-integrated photoluminescence (PL) spectra recorded for various excitation intensity for GaN epilayers grown over $r$-plane (a), $a$-plane (b), and c-plane (c) sapphire substrate. Luminescence spectra of GaN epilayers are formed by high-density electron-hole plasma (EHP) emission that occurs under intense photoexcitation with the excitonic states screened by the nonequilibrium carrier system [7, 8]. All spectra consist of one broad emission band. The EHP band is peaked in the vicinity of the band gap energy $(\approx 3.4 \mathrm{eV})$, it dominates in the emission in a broad range of excitation intensities. For the highest excitation $I_{\text {exc }}>10 \mu \mathrm{J}$, the spectra of polar samples are modified due to stimulated emission (at $3.3 \mathrm{eV}$ ). Figures 1d, e and f show PL spectra of AlGaN/GaN MQWs grown over corresponding substrates. Spectra are slightly broadened, due to inhomogeneity 

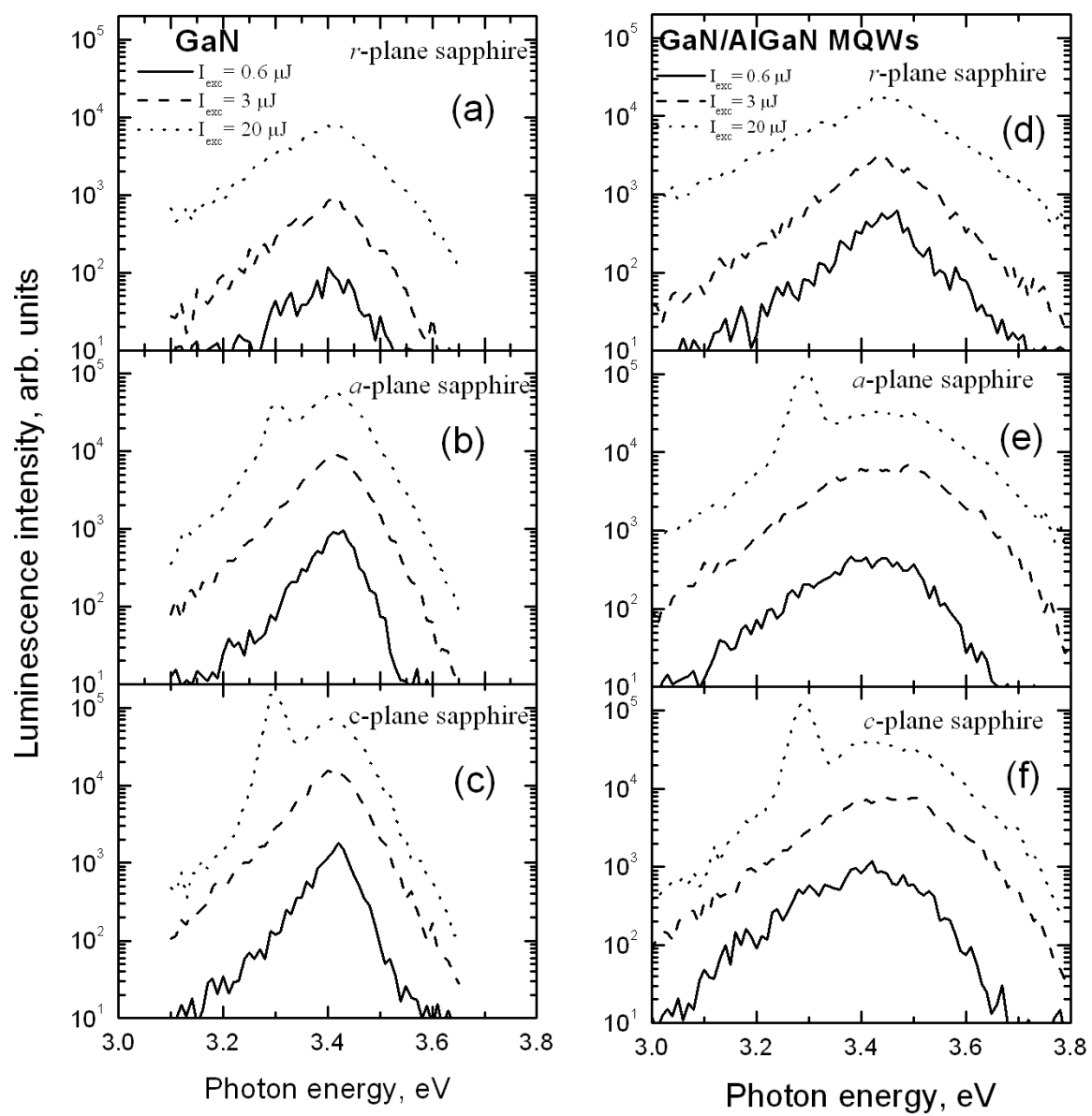

Fig. 1. Time-integrated luminescence spectra of GaN epilayers and AlGaN/GaN MQWs grown over various crystal planes of sapphire substrate: $r$-plane (a) and (d), $a$-plane (b) and (e), and c-plane (c) and (f), recorded for various excitation intensities.

of the MQWs, and blueshifted, due to spatial confinement effect, as compared to corresponding ones of GaN epilayer emission (Figs. 1a, b and c).

Figure 2 shows excitation energy dependence of the PL peak position (a) and (c) and the spectrally integrated intensity (b) and (d) taken from Fig. 1. Points obtained for low cw excitation are shown for comparison (indicated in Fig. 2 by arrows). Excitation induced shrinkage of the band gap (up to $20 \mathrm{meV}$ ) for GaN epilayers is observed in Fig. 1a. Small variations in energy position evident at low excitation are due to different piezoelectric stress in samples grown over different planes of sapphire substrate. These variations diminish with increased carrier density. PL intensity is seen to differ significantly in different samples (Fig. 2b). Namely, the GaN grown over $r$-plane sapphire exhibit a decrease in 

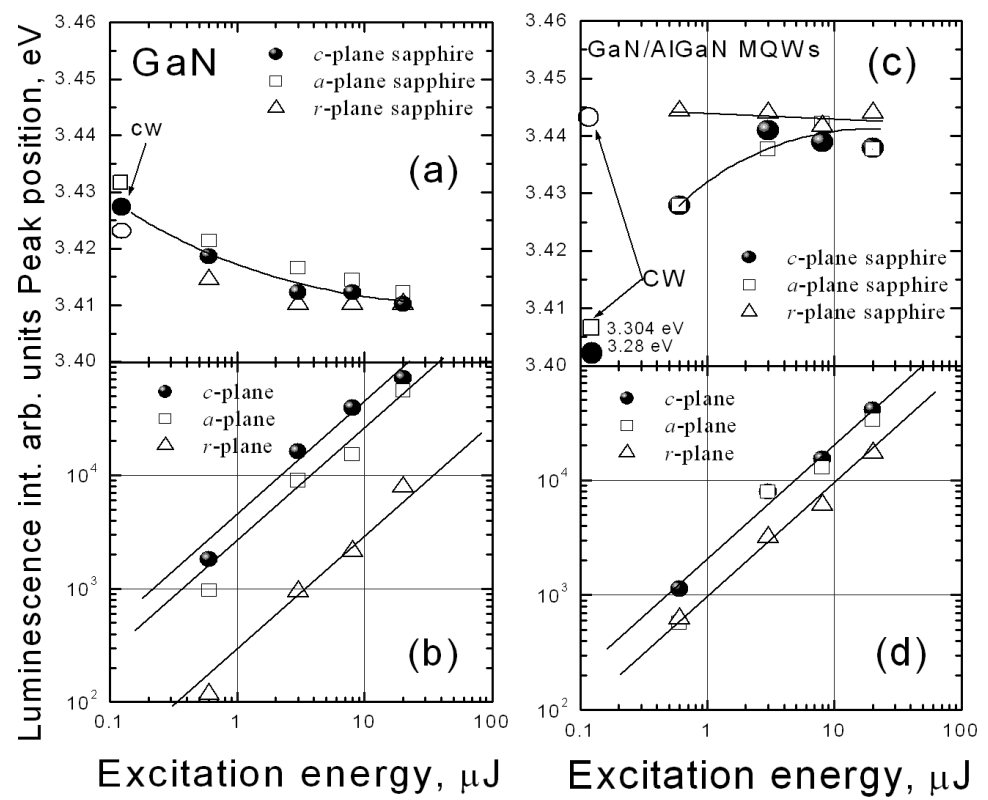

Fig. 2. Excitation energy dependence of the luminescence peak position (a) and (c) and spectrally integrated intensity (b) and (d) obtained from Fig. 1. Points - experiment, lines - guide for eyes. Points obtained for low cw excitation are shown for comparison (indicated by arrows).

EHP luminescence intensity by about 20 times in comparison with the conventional $c$-plane GaN epilayers grown over $c$-plane sapphire, whereas the $a$-plane sapphire grown GaN shows almost the same PL intensity. Luminescence efficiency well correlates with the luminescence decay time obtained at high photoexcitation of $1 \mathrm{~mJ} / \mathrm{cm}^{2}$. Polar samples grown over $c$ - and $a$-planes show luminescence decay time $\tau_{\mathrm{LU}}=42 \mathrm{ps}$, while nonpolar epilayer $\tau_{\mathrm{LU}} \leq 10 \mathrm{ps}$. Decrease in luminescence efficiency is due to nonradiative traps that are most likely related to threading dislocations coming from the substrate [8].

In MQWs grown on polar planes, a blueshift of the luminescence band with increasing the excitation energy is evident from Fig. 2c (from about $3.30 \mathrm{eV}$ at low excitation to $3.44 \mathrm{eV}$ at high excitation intensity), indicating on screening of built-in field by free carriers (the nonequilibrium-carrier density is of the order of $\left.10^{19} \mathrm{~cm}^{-3}\right)$. Whereas in nonpolar $r$-plane grown MQWs, the luminescence band maintained an almost constant peak position $(3.44 \mathrm{eV})$. Full screening of built-in field was observed at the excitation densities higher than $0.3 \mathrm{~mJ} / \mathrm{cm}^{2}$. An almost linear dependence of PL intensity on excitation intensity (Fig. 2d) is in line with this observation. Under such conditions, separation of electron-hole wave functions in quantum wells is negligible and the quality of the structure can be characterized by luminescence efficiency and carrier lifetime. The estimated 
luminescence decay time is $20 \mathrm{ps}, 29 \mathrm{ps}$, and $35 \mathrm{ps}$ for MQWs grown over $r$-, $a$-, and $c$-plane sapphire substrate, respectively. Thus, although formation of MQW structure reduces the number of threading dislocations, the quality of the nonpolar structures are still worse than that of conventional $c$-plane grown MQWs.

In conclusion, formation of nonpolar AlGaN/GaN structures on $r$-plane of sapphire substrate requires suppressing of the lattice mismatch related nonradiative recombination. In particular, epitaxial lateral overgrowth technique can be the method of choice that can reduce nonradiative recombination in field-free nitride heterostructures.

\section{Acknowledgments}

The research at Vilnius University was partially supported by the Lithuanian State Science and Education Foundation under COST529 program and European Commission supported SELITEC center contract No. G5MA-CT-200204047. A.Ž. acknowledges the Lithuanian Ministry of Education and Science for his fellowship.

\section{References}

[1] S. Nakamura, G. Fasol, The Blue Laser Diode: GaN Based Light Emitters and Lasers, Springer, Berlin 1997.

[2] P. Waltereit, O. Brandt, M. Ramsteiner, R. Uecker, P. Reiche, K.H. Ploog, J. Cryst. Growth 218, 143 (2000).

[3] M.D. Craven, P. Waltereit, J.S. Speck, S.P. DenBaars, Appl. Phys. Lett. 84, 496 (2004).

[4] Y.J. Sun, O. Brandt, S. Cronenberg, S. Dhar, H.T. Grahn, K.H. Ploog, P. Waltereit, J.S. Speck, Phys. Rev. B 67, 041306 (2003).

[5] W.H. Sun, J.W. Yang, C.Q. Chen, J.P. Zhang, M.E. Gaevski, E. Kuokstis, V. Adivarahan, H.M. Wang, Z. Gong, M. Su, M.A. Khan, Appl. Phys. Lett. 83, 2599 (2003).

[6] C.Q. Chen, J.W. Yang, H.M. Wang, J.P. Zhang, V. Adivarahan, M. Gaevski, E. Kuokstis, Z. Gong, M. Su, M.A. Khan, Jpn. J. Appl. Phys. 42, L640 (2003).

[7] F. Binet, J.Y. Duboz, J. Off, F. Scholz, Phys. Rev. B 60, 4715 (1999).

[8] S. Juršènas, G. Kurilčik, N. Kurilčik, A. Žukauskas, P. Prystawko, M. Leszczynski, T. Suski, P. Perlin, I. Grzegory, S. Porowski, Appl. Phys. Lett. 78, 3776 (2001). 